\title{
Erratum to: A Therapeutic Insight of Niacin and Coenzyme Q10 against Diabetic Encephalopathy in Rats
}

\author{
Tarek K. Motawi ${ }^{1} \cdot$ Hebatallah A. Darwish ${ }^{1}$ • Manal A. Hamed ${ }^{2}$. \\ Nagy S. El-Rigal ${ }^{2}$ - Asmaa F. Aboul Naser ${ }^{2}$
}

Published online: 20 March 2017

(C) Springer Science+Business Media New York 2017

\section{Erratum to: Mol Neurobiol (2017) 54 (3): 1601-1611 DOI 10.1007/s12035-016-9765-x}

In some parts of this article, some mistakes were detected. With these, the authors would like to correct them as follows: In Abstract line 17, it should be "acetylcholinesterase (AchE)" instead of acetylcholine (Ach).

In Experimental Design: The first sentence should be corrected to "...seventy two rats were divided into nine groups (eight rats each).

In Biochemical Determinations, paragraph 2 line 4, the sentence "The total antioxidant Capacity..." should be deleted. Also in the third paragraph at line 1, instead of acetylcholine (Ach) it should be written as "acetylcholinesterase (AchE)".

The online version of the original article can be found at http://dx.doi. org/10.1007/s12035-016-9765-x

Manal A. Hamed

manal_hamed@yahoo.com

Biochemistry Department, Faculty of Pharmacy, Cairo University, Cairo, Egypt

2 Therapeutic Chemistry Department, National Research Centre, 33 El-Bohouth St, Dokki, Giza 60014618, Egypt
In Biochemical Results, Page 5 at third paragraph, line 1, it should be AchE instead of Ach.

In Statistical Analysis, first line, it was written as "ten" but it should be "eight".

And lastly in Fig. 2, The Y-axis is should be Acetylcholinesterase as presented below.
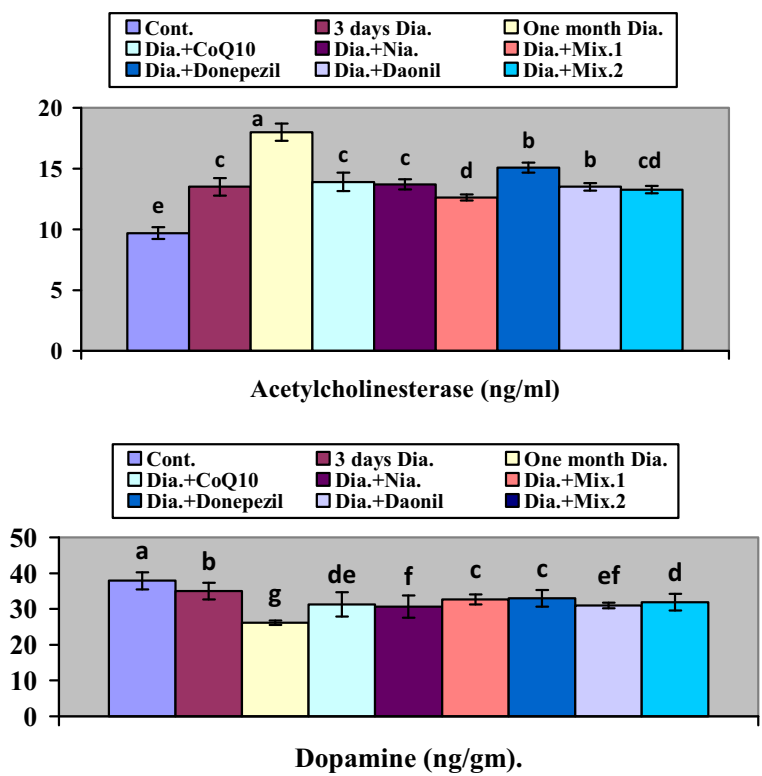

- Data are mean $\pm \mathrm{SD}$ of eight rats in each group.

- Unshared superscript letters are significant values between groups at $p<0.0001$.

Fig. 2: Therapeutic effects of different drugs on neurotransmitter levels. 